\title{
WHAT IS THE REAL PUBLIC HEALTH SIGNIFICANCE OF IRON DEFICIENCY AND IRON DEFICIENCY ANAEMIA IN CROATIA? \\ A POPULATION-BASED OBSERVATIONAL STUDY ON PREGNANT WOMEN AT EARLY PREGNANCY FROM EASTERN CROATIA
}

\author{
Ines Banjari, Daniela Kenjerić, Milena L. Mandić \\ Department of Food and Nutrition Research, Faculty of Food Technology, University of Osijek, Osijek, Croatia
}

\section{SUMMARY}

Aim: Studies imply that significance of iron deficiency (ID) and iron deficiency anaemia (IDA) for pregnancy outcomes is especially highlighted in the early pregnancy. Prevalence around the world varies widely, however, no data is available up to date for Croatia or neighbouring countries. Therefore, the objective was to determine the prevalence of ID and IDA among pregnant women from Croatia at the first trimester. Also, the aim was to compare two criterions; the World Health Organization (WHO) one and the clinical one.

Methods: Randomised observational population based study was set up and 265 pregnant women at the first trimester were enrolled.

Results: Based on the WHO criteria, $17.7 \%$ on haemoglobin basis and $18.5 \%$ on haematocrit basis had either ID or IDA. Clinical criteria showed that even $32.8 \%$ had either ID or IDA (transferrin saturation $<20.0 \%$ ). The WHO criterion shows less sensitivity, especially in detecting less severe stages of IDA.

Conclusions: Regardless of the criteria used, ID and IDA present a mild to moderate public health problem in pregnant women population. This high share of pregnant women who are starting their pregnancy as iron deficient, presents a potentially high risk for the pregnancy outcomes, especially in terms of a newborn, and it is fully justified to treat them as diseases of public health significance.

Key words: iron deficiency, iron deficiency anaemia, pregnant women, early pregnancy, Croatia

Address for correspondence: I. Banjari, University of Osijek, Faculty of Food Technology, Department of Food and Nutrition Research, F. Kuhača 20, HR-31000 Osijek, Croatia. E-mail: ines.banjari@ptfos.hr

\section{INTRODUCTION}

Iron deficiency (ID) and iron deficiency anaemia (IDA) are major nutritional problems throughout the world and present a problem of major public health significance (1). ID without anaemia is more common and indicates depleted iron stores that are still sufficient to maintain a normal level of haemoglobin $(2,3)$. According to the World Health Organization (WHO), the frequency of ID in developing countries is about 2.5 times that of anaemia (2). This gradient progression from ID to IDA is one of the main characteristics of the disease, which is, if untreated, progressive with large overall impacts $(3,4)$. The incidence of anaemia in both, industrialized and developing countries is especially high during pregnancy and lactation due to an increase need for iron $(1,5)$. However, many of these women were already anaemic at the time of conception $(1,6,7)$, and estimated prevalence of anaemia is $43 \%$ in non-pregnant women in developing countries and $12 \%$ in wealthier regions (2). ID and IDA often develop during the later stages of pregnancy even in women who enter pregnancy with relatively adequate iron stores $(6,8)$. The problem is recognized and listed as one of the main goals in prevention measures of public health significance by the Croatian Food and Nutrition Policy from 1999 (9). Still, to the authors' best knowledge, no data is available to wider public or scientists regarding the prevalence or incidence of ID or IDA in pregnant women in Croatia, or in other neighbouring countries (e.g. Serbia, Macedonia, Slovenia, Bosnia and Herzegovina, Montenegro, and Hungary).

WHO defines anaemia as haemoglobin level below $110 \mathrm{~g} / 1$ for pregnant women, or haematocrit level below 0.330 1/1 (2, 10). This criterion has been widely argued, since it is known that haemoglobin itself cannot be considered as the only indicator of IDA. Therefore, clinical interpretation is useless unless iron binding capacity values, i.e. transferrin saturation percentage, are available at the same time $(11,12)$. In IDA unsaturated iron binding capacity (UIBC) and total iron binding capacity (TIBC) are increased while usual transferrin saturation, which is normally $20-50 \%$, drops below $15.0 \%$. So, for screening purpose and clinical decision, WHO recommends besides haemoglobin and haematocrit determination of either serum ferritin or transferrin saturation $(2,10,13)$. Even though serum ferritin is the golden standard for iron stores it is also an indicator of inflammation and therefore can give false positive results, but it is also not a standard 
screening procedure used among practitioners (i.e. gynaecologists) in Croatia. Depending on the prevalence of anaemia in a certain population it can be categorised as a problem of public health significance (2).

Although the maternal red-cell mass and plasma volume both increase during gestation, they do not do so simultaneously, therefore it is difficult to distinguish physiologic anaemia from IDA in the late pregnancy. It is thus becoming clear that the best time to detect any risk associated with maternal anaemia may be early in pregnancy (14).

It is well known that maternal anaemia induced by ID adversely affects pregnancy outcome. The effects include low birth weight infants, preterm delivery, up to labour complications, and higher rates of caesarean section $(6,7,15-19)$. The risk is higher as the haemoglobin is lower, especially if ID and IDA were present at early pregnancy $(6,7,14-18)$.

With the above in mind, the main objective was to determine the prevalence of ID and IDA among pregnant women at early pregnancy from eastern Croatia region. The secondary objective was to observe possible difference in the prevalence by using different criteria; the WHO and clinical ones. Also, if the difference exists, to determine which criterion is more reliable and gives more reliable data when the physiology and stages in the development of IDA are considered.

\section{MATERIALS AND METHODS}

For the randomized sample, inclusion criteria was healthy pregnancy within 12 weeks of gestation (i.e. 1st trimester), followed in two general gynaecologist office from area of city Osijek, Eastern Croatia. Early pregnancy was selected since statistical data for the past several years on the research area show that for around $88 \%$ of pregnancy is confirmed within 12 weeks of gestation $(20,21)$. The inclusion criterion was healthy status, not having any special dietary regime (e.g. coeliac disease), without any medicament treatment.

Two hundred and sixty five pregnant women were enrolled during one year period and monitored (throughout gestation to labour) from 2010 till the end of 2011. The study was approved by the Ethical committee of the Faculty of Food Technology Osijek; an informed consent was obtained from all participating pregnant women.

General data regarding age, education level, incomes, earlier pregnancies, specific conditions, smoking habits, and supplement use were collected through a short questionnaire developed for this study. Basic data were collected at the first interview when the pregnancy was confirmed, i.e. within the first 12 weeks of gestation. Recruited population is by all demographic and socioeconomic characteristics representative for the overall population of pregnant women from encompassed area of city Osijek (20-22), and presents $17.7 \%$ of the total population of pregnant women for the study period.

Blood samples were collected and analyzed in medicalbiochemical laboratory for iron status on OLYMPUS AU400, OLYMPUS AU680 and Coulter LH750 Analyzer. Iron blood status was covered by the standard blood analysis parameters: erythrocytes, haemoglobin, haematocrit, mean corpuscular volume (MCV), mean cellular haemoglobin (MCH), mean corpus- cular haemoglobin concentration (MCHC), serum iron (SI), and unsaturated iron binding capacity (UIBC), while total iron binding capacity (TIBC) and transferrin saturation (TfS) were calculated $(\mathrm{TIBC}=\mathrm{UIBC}+\mathrm{SI} ; \mathrm{TfS}=(\mathrm{SI} / \mathrm{TIBC}) \times 100 \%)$.

Prevalence of ID and anaemia due to iron deficiency were determined on the basis of both WHO criteria $(2,10)$ and clinical reference levels of other iron status parameters like serum iron, and transferrin saturation $(1,3,23)$. Level used for the classification of ID and IDA as public health problem was based on the WHO criteria (2).

Statistical analysis was performed with software tool Statistica 8.0 (StatSoft, Tulsa, Oklahoma, USA), at significance level $\mathrm{p}=0.05$. Normality of data distribution was tested by the nonparametric Kolmogorov-Smirnov test for the comparison of medians and arithmetic mean, and histograms plotting. T-test and Wilcoxon test were used to test two dependent variable groups, depending on the normality of the data.

\section{RESULTS}

Distribution of the participating pregnant women throughout general characteristics is shown in Table 1. Mean age was 28.9 years and high percentage of older pregnant women was noted; $10.9 \%$ of the participating pregnant women fell into the high risk group of $35+$ years of age. Most of the participating pregnant women were primiparae (50.6\%), and biparae (34.3\%). Multiple pregnancies were present in $15.1 \%$ of women. Lifestyle habits of pregnant women can be considered as favourable. Dietary supplementation was abundant (82.6\% of pregnant women). Out of 136 women who had smoked before pregnancy $(51.3 \%), 99$ (72.8\%) decided to stop smoking during pregnancy.

Observed blood parameters related to iron status in the study population are shown in Table 2. Ranges give insight in the wide distribution of iron blood status among women, especially if the reference values (the lowest value for example for MCV, SI and TfS or the highest value for TIBC) were considered.

The WHO criteria for haemoglobin level show that the total prevalence of ID and IDA among pregnant women is $17.7 \%$ (Table 3). Out of that, $4.5 \%$ have IDA, while $13.2 \%$ have ID.

Table 1. Distribution of the participating pregnant women $(N=265)$ based on general characteristics

\begin{tabular}{|c|c|c|c|c|}
\hline Characteristic & Average & Distribution & $\mathrm{n}$ & $\%$ \\
\hline \multirow{3}{*}{ Age } & \multirow{3}{*}{$28.9 \pm 4.9$} & $\leq 19 \mathrm{yr}$ & 5 & 1.9 \\
\hline & & $20-34 \mathrm{yr}$ & 231 & 87.2 \\
\hline & & $\geq 35 \mathrm{yr}$ & 29 & 10.9 \\
\hline \multirow{2}{*}{$\begin{array}{l}\text { Taking } \\
\text { supplements }\end{array}$} & & no & 46 & 17.4 \\
\hline & & yes & 219 & 82.6 \\
\hline \multirow{3}{*}{$\begin{array}{l}\text { Smoking in } \\
\text { pregnancy }\end{array}$} & & never & 129 & 48.6 \\
\hline & & before pregnancy & 99 & 37.4 \\
\hline & & continued smoking & 37 & 14.0 \\
\hline \multirow{3}{*}{ Parity } & \multirow{3}{*}{$1.7 \pm 0.9$} & primiparae & 134 & 50.6 \\
\hline & & biparae & 91 & 34.3 \\
\hline & & multiparae & 40 & 15.1 \\
\hline
\end{tabular}


Table 2. Observed iron blood parameters related to iron status of the participating pregnant women $(N=265)$ expressed as mean values and ranges

\begin{tabular}{|c|c|c|}
\hline Parameter & Mean \pm SD & Range \\
\hline Erythrocytes $\left(\mathrm{E} ; \times 10^{12} / \mathrm{l}\right)$ & $4.31 \pm 0.35$ & $3.36-5.31$ \\
\hline Haemoglobin (Hg; g/l) & $127.2 \pm 9.9$ & $89-155$ \\
\hline Haematocrit (Hct; I/l) & $0.382 \pm 0.028$ & $0.299-0.466$ \\
\hline $\begin{array}{l}\text { Mean corpuscular volume } \\
\left(\mathrm{MCV} ; \times 10^{15} /\right)\end{array}$ & $88.2 \pm 4.6$ & $72.0-105.6$ \\
\hline $\begin{array}{l}\text { Mean corpuscular haemoglobin } \\
\left(\mathrm{MCH} ; \times 10^{12} / \mathrm{g}\right)\end{array}$ & $29.5 \pm 1.8$ & $20.8-35.6$ \\
\hline $\begin{array}{l}\text { Mean corpuscular haemoglobin } \\
\text { concentration (MCHC; g/l) }\end{array}$ & $332.7 \pm 9.5$ & $290-363$ \\
\hline Serum iron (Sl; $\mu \mathrm{mol} / \mathrm{l})$ & $16.7 \pm 7.1$ & $3.3-42.5$ \\
\hline $\begin{array}{l}\text { Unsaturated iron binding capacity } \\
\text { (UIBC; } \mu \mathrm{mol} / \mathrm{l})\end{array}$ & $46.5 \pm 13.8$ & $18.0-92.3$ \\
\hline $\begin{array}{l}\text { Total iron binding capacity } \\
\text { (TIBC; } \mu \mathrm{mol} / \mathrm{l})\end{array}$ & $63.2 \pm 10.5$ & $43.0-96.4$ \\
\hline Transferrin saturation (TfS; \%) & $27.5 \pm 12.9$ & $4.3-66.4$ \\
\hline
\end{tabular}

Table 3. Distribution of the participating pregnant women $(N=265)$ based on $W H O$ and clinical criteria, presented as reference ranges for haemoglobin, haematocrit, serum iron and transferrin saturation

\begin{tabular}{|c|c|c|c|}
\hline Parameter & Reference range & $\mathrm{n}$ & $\%$ \\
\hline \multirow{3}{*}{$\begin{array}{l}\text { Haemoglobin } \\
(\mathrm{Hg} ; \mathrm{g} / \mathrm{l})\end{array}$} & $<110$ & 12 & 4.5 \\
\hline & 110-118 & 35 & 13.2 \\
\hline & 119-158 & 218 & 82.3 \\
\hline \multirow{3}{*}{$\begin{array}{l}\text { Haematocrit } \\
(\mathrm{Hct} ; \mathrm{l} / /)\end{array}$} & $\leq 0.330$ & 10 & 3.8 \\
\hline & $0.331-0.355$ & 39 & 14.7 \\
\hline & $0.356-0.470$ & 216 & 81.5 \\
\hline \multirow{3}{*}{$\begin{array}{l}\text { Serum iron } \\
(\mathrm{Sl} ; \mu \mathrm{mol} / \mathrm{l})\end{array}$} & $\leq 8.0$ & 37 & 14.0 \\
\hline & $8.1-30.0$ & 220 & 83.0 \\
\hline & $\geq 30.1$ & 8 & 3.0 \\
\hline \multirow{3}{*}{$\begin{array}{l}\text { Transferrin saturation } \\
\text { (TfS; \%) }\end{array}$} & $<15.0$ & 49 & 18.5 \\
\hline & $15.0-19.9$ & 38 & 14.3 \\
\hline & $\geq 20.0$ & 178 & 67.2 \\
\hline
\end{tabular}

On the other hand, if the WHO criterion for haematocrit level is taken, than the total prevalence is $18.5 \%$, out of which $3.8 \%$ have IDA while $14.7 \%$ have ID. The difference in the prevalence of ID and IDA in pregnant women depending on both WHO criteria is shown in Fig. 1.

For the clinical interpretation other iron blood parameters are necessary, and SI as well as UIBC and TIBC, i.e. TfS are probably the most important. In IDA serum iron is low, and the results show that $14.0 \%$ of pregnant women have low SI (Table 3). Since TfS includes other iron blood parameters, including SI, it can provide more accurate information on the status of iron. Therefore, the prevalence of ID and IDA based on TfS is in total $32.8 \%$, or more accurately, $18.5 \%$ of pregnant women have IDA and $14.3 \%$ ID (Table 3 ). In other words, this is a more sensitive parameter, while haemoglobin shows high level of plasticity. This difference in sensitivity is shown in Fig. 2.

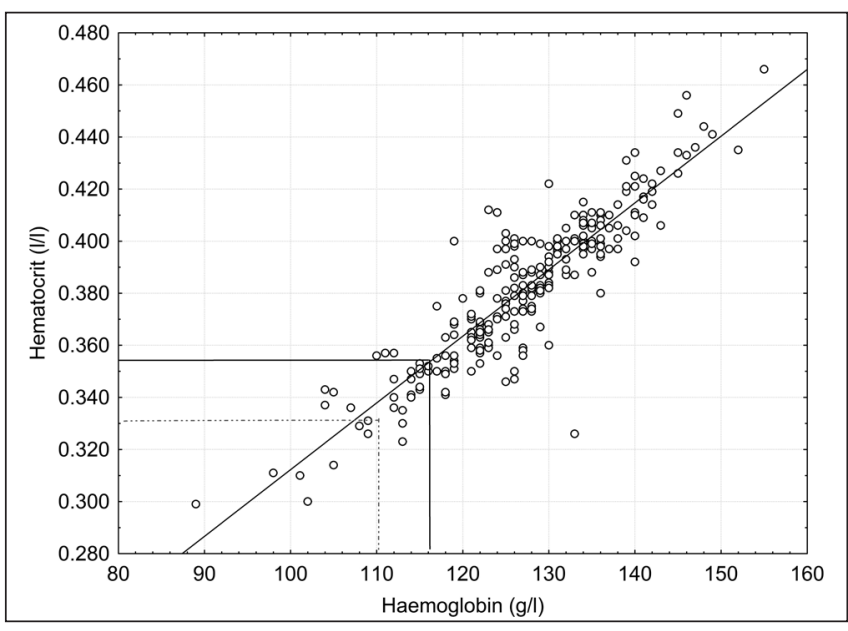

Fig. 1. The diference in prevalence of pregnant women with ID (full lines) and IDA (dashed lines) on the basis of the WHO criteria for both haemoglobin and haematocrit values in the participating pregnant women $(N=265)$ at early pregnancy.

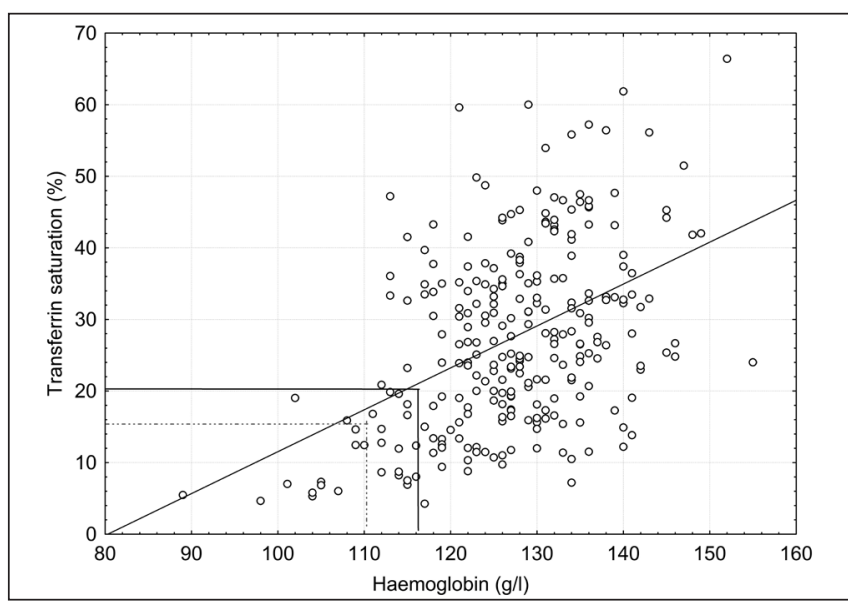

Fig. 2. Prevalence of ID (full lines) and IDA (dashed lines) based on haemoglobin and transferrin saturation in the participating pregnant women at the first trimester $(N=265)$.

On the basis on haemoglobin values in early pregnancy and WHO cutt-off value of $\leq 110 \mathrm{~g} / \mathrm{l}$, pregnant women were distributed in one of the three categories. The first one $(\mathrm{Hg}<110 \mathrm{~g} / \mathrm{l})$ presents IDA women, $\mathrm{Hg}$ 110-118 g/l presents ID women and the third one with $\mathrm{Hg} 119-158 \mathrm{~g} / \mathrm{l}$ are women with normal iron status at early pregnancy. The change of all other observed blood parameters for iron status are shown in Fig. 3. Haematocrit is constant in all three haemoglobin categories. Other parameters show consistency with physiology and nature of the disease. The lowest values are found in the lowest haemoglobin category, for SI, MCV and TfS. This group also has the highest absolute values of UIBC and TIBC.

Response of iron blood parameters based on the transferrin saturation is presented in Fig. 4. Based on the cut-off value for TfS of $<15.0 \%$ of pregnant women were rearranged in three categories. The first category represents IDA women (TfS $<15.0 \%$ ), the second represents ID women (TfS 15.0-19.9\%) and the third one with TfS $\geq 20.0 \%$ represents women with normal iron status at early pregnancy. Again, the same trend in change of iron blood parameters can be noted. No significant change in absolute change of haematocrit between these three categories can be noted. The lowest values are found in the lowest TfS category, for $\mathrm{Hg}$, SI, and 


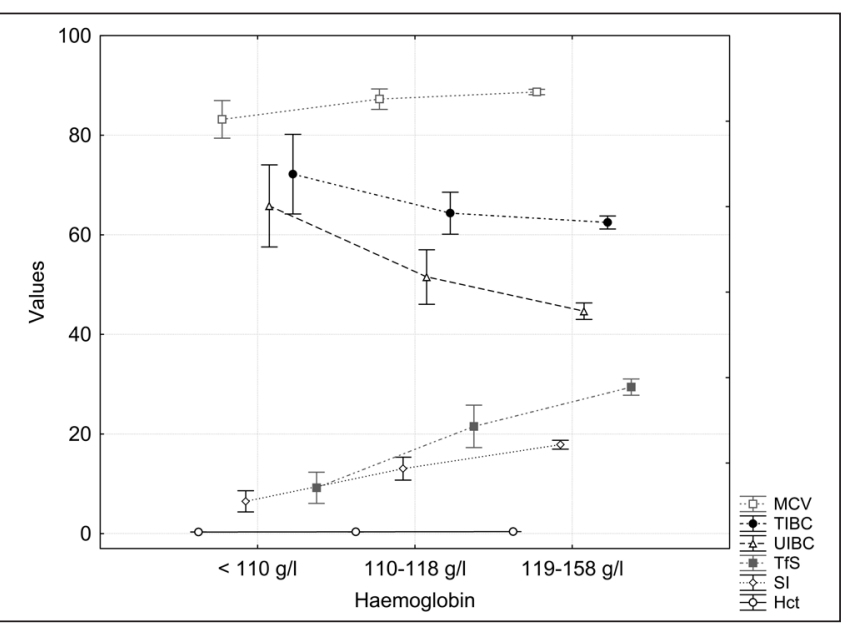

Fig. 3. Response of iron blood status parameters expressed as absolute change in value depending on haemoglobin value of the participating pregnant women $(N=265)$ at early pregnancy.

MCV. This group also has the highest absolute values of UIBC and TIBC. Additionaly, confidence interval shows much better values if TfS is taken as a cut-off value for dividing pregnant women into different iron status categories.

\section{DISCUSSION}

Estimates from the WHO report say that from $35 \%$ to $75 \%$ (56\% on average) of pregnant women in developing countries, and $18 \%$ of pregnant women in industrialized countries are anaemic, with half suffering from iron deficiency anaemia (2). Our data show that although average haemoglobin value is within the normal range (127.2 $\pm 9.9 \mathrm{~g} / \mathrm{l})$ (Table 2$), 17.7 \%$ of the pregnant women start their pregnancy with low haemoglobin (below 118 $\mathrm{g} / \mathrm{l})$, or haematocrit $(18.5 \%$ below $0.355 \mathrm{l} / \mathrm{l})$. This prevalence of ID and IDA among pregnant women at the 1st trimester is in accordance with the WHO estimates for industrialized countries (2), while ID and IDA among pregnant women from Croatia can be categorised as a mild public health problem. Our findings confirm higher frequency of ID rather than IDA. ID in Croatia is 3.8 times

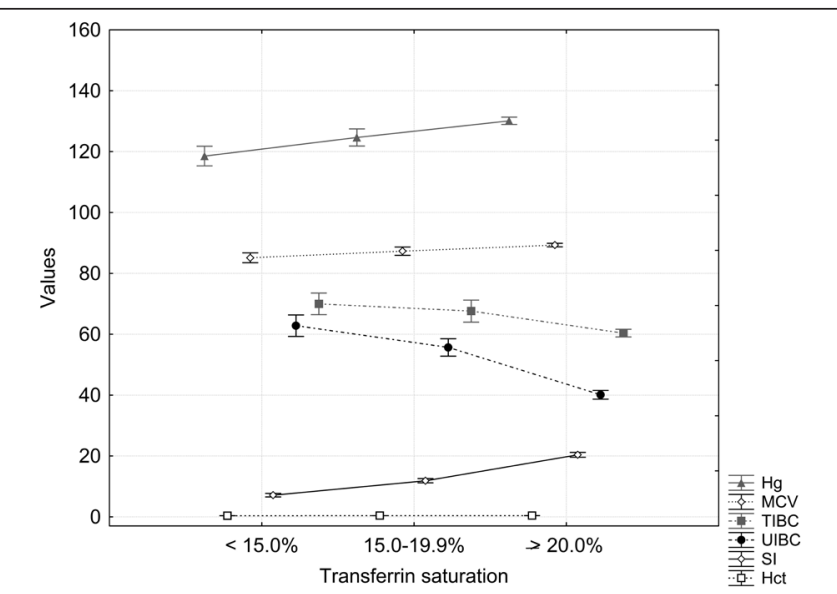

Fig. 4. Response of iron blood status parameters expressed as absolute change in value depending on transferrin saturation value of the participating pregnant women $(N=265)$ at early pregnancy. more frequent at early pregnancy, which is higher than previously assumed by the WHO (2). Significance of these findings lies in the fact that these women are at increased risk of developing IDA in the later stages of pregnancy $(6,8,24)$.

Despite being the main criteria of the WHO, haemoglobin has been widely argued for its plasticity and robustness in recognizing less severe changes in iron status $(2,11,13,19)$. So, for more precise clinical diagnosis more parameters on iron blood status are needed (11-13). ID is usually indicated by reduced MCH and reduced MCV. In IDA serum iron is low and the level of transferrin measured as TIBC is elevated, resulting in reduced transferrin saturation $(2,10,13,19,25-28)$. When serum iron concentration of the studied population is considered, $14.0 \%$ of pregnant women have SI below $8.0 \mu \mathrm{mol} / 1$, but when transferrin saturation is observed, $32.8 \%$ of the participating pregnant women at the 1st trimester have values below $20.0 \%$ which indicates ID and/ or IDA $(4,13)$. Having in mind the complexity of the TfS value itself, which includes both SI and TIBC, higher sensitivity was expected and proven by higher prevalence. Higher sensitivity of TfS vs Hg is also shown in regard to better confidence intervals for other observed parameters of iron blood status (Fig. 3 and 4). Considering the necessity of early diagnosis of any stage of iron deficiency, we, like many other authors before $(11,13)$, recommend the use of this criterion. This is highly recommended in the case of general gynaecology follow where serum ferritin is not a necessity, and if the financial issue exist. These are all the case in Croatia, as well as in many neighbouring developing countries (e.g. Serbia, Bosnia and Herzegovina, Montenegro, and Macedonia). On the basis of TfS criteria the prevalence of ID and IDA in pregnant women from Croatia presents a moderate public health problem.

Early pregnancy has been suggested as the best period for anaemia detection among pregnant women (8), and the presented results indicate that despite of the criteria used, the percentage of real anaemia and depleted iron stores among pregnant women at early pregnancy in Croatia is quite high.

In terms of iron status it is necessary to pay attention to the lifestyle and nutritional habits as well. Some studies imply that pregnant women tend to change these habits for healthier ones (30). Surely, among important aspects for lifestyle changes are supplementation, and change in smoking habits. Their importance lies in their relation either to iron blood status or the physiology of the disease itself.

Dietary supplementation in pregnancy is highly recommended $(1,5,7)$ because of the increased needs during pregnancy that can be hardly satisfied only throughout regular diet. Especially since the dietary intake of iron among pregnant women has been proven to be low $(5,17,29,30,31)$ and of low bioavailability $(31,32)$. Supplementation was frequently noted among the participating pregnant women from Croatia (82.6\%). Contribution to iron intake is expected to be influenced, resulting in approved iron blood status. A study by Scanlon et al. (18) showed that the prevalence of anaemia (based on haemoglobin level) among iron-supplemented pregnant women participating in public health nutrition programmes is approximately $8 \%$ at the 1 st trimester. Still, despite the abundance of supplementation we determined much higher prevalence of anaemic and/or iron deficient pregnant women from Croatia at the 1 st trimester.

Smoking has been associated with the increased risk for spontaneous abortion, especially during the 1 st trimester, reduced birth 
weight, and perinatal mortality $(33,34)$. As for the studied population from Croatia we found that $27.2 \%$ of smokers continue to smoke during pregnancy regardless of doctor's recommendations and knowledge about its impact on child's health. Also, smoke and ID and IDA in combination have even greater effect in terms of negative impact on pregnancy outcomes, especially in terms of low birth weight and preterm delivery $(33,34)$. Pregnancy is the period in every woman's life when she is more willing to change her dietary as well as lifestyle habits for healthier ones (30), which is a trend seen in the studied population from Croatia, e.g. $72.8 \%$ of smokers decided to stop smoking during pregnancy.

Our findings on supplements use and readiness for smoking cessation confirm earlier results on women's willingness to change their lifestyle habits for healthier ones during pregnancy.

The level of impact that poor iron blood status has on pregnancy outcomes emphasizes the need for close monitoring of women throughout pregnancy. Scholl et al. (16) found that only iron-deficiency anaemia during the 1 st and 2nd trimester of pregnancy increased a woman's risk for preterm delivery and delivering a low birth weight infant, providing strong evidence that IDA is associated with preterm birth. Scanlon et al. (18) showed that the risk of preterm birth was increased in women with low haemoglobin level in the 1 st and 2 nd trimester. The risk depended on the severity of haemoglobin deficit. Additionally, they found that the rates of preterm delivery and low birth weight, but not small for gestation births, were increased in women who had anaemia early in pregnancy (18).

Overall, even though ID presents the less severe stage in the anaemia development, numerous studies have shown that it presents a significant risk factor in pregnancy, especially in terms of the foetal programming $(35,36)$, and that it should not be neglected by the practitioners for its underlying consequences.

\section{CONCLUSIONS}

Prevalence of iron deficiency and iron deficiency anaemia in pregnant women from Croatia at early pregnancy is in accordance with the WHO estimations. Depending on the criteria used they can be categorised as a mild or moderate public health problem. Robustness of haemoglobin, a referent criterion by the WHO, was again proven as a preferable criterion, so we recommend the use of transferrin saturation, especially for clinical purposes. The results are important not just for Croatia but for all neighbouring countries, since these population based data on the prevalence of ID or IDA on pregnant women present good starting point in different national prevention programmes as well as in clinical practice.

\section{Acknowledgements}

The research has been conducted as a part of scientific project „Nutrition and lifestyle in health protection“, financed by the Ministry of Science, Education and Sports of Croatia. No other sources of financing has been used.

Special thanks go to medical staff from the Gynaecologists' offices and Medical-biochemical laboratory as well as to all pregnant women who participated in this study.

\section{Conflict of Interests}

None declared

\section{REFERENCES}

1. Zimmermann MB, Hurrell RF. Nutritional iron deficiency. Lancet. 2007 Aug 11;370(9586):511-20.

2. UNICEF; UNU; WHO. Iron deficiency anaemia: assessment, prevention, and control. Geneva: WHO; 2001.

3. Adamson JW. Iron deficiency and other hypoproliferative anemias. In: Fauci AS, Braunwald E, Kasper DL, Hauser SL, Longo DL, Jameson JL, et al. editors. Harrison's principles of internal medicine. 17th ed. New York: McGraw-Hill; 2008. p. 628-34.

4. Heidemann $\mathrm{BH}$. Changes in maternal physiology during pregnancy. Update in Anaesthesia. 2005;(20):21-4.

5. Lee JI, Kang SA, Kim SK, Lim HS. A cross sectional study of maternal iron status of Korean women during pregnancy. Nutr Res. 2002;22(12):1377-88.

6. Allen LH. Anemia and iron deficiency: effects on pregnancy outcome. Am J Clin Nutr. 2000 May;71(5 Suppl):1280S-4S.

7. Milman N, Bergholt T, Eriksen L, Byg KE, Graudal N, Pedersen P, et al. Iron prophylaxis during pregnancy - how much iron is needed? A randomized dose-response study of 20-80 mg ferrous iron daily in pregnant women. Acta Obstet Gynecol Scand. 2005 Mar;84(3):238-47.

8. Scholl TO. Iron status during pregnancy: setting the stage for mother and infant. Am J Clin Nutr. 2005 May;81(5 Suppl):1218S-1222S.

9. Ministry of Health of the Republic of Croatia; Croatian National Institute of Public Health. Croatian Food and Nutrition Policy. Zagreb: Croatian National Institute of Public Health; 1999. (In Croatian.)

10. World Health Organization; Centers for Disease Control and Prevention. Assessing the iron status of populations. Report of a Joint World Health Organization/Centers for Disease Control and Prevention Technical Consultation on the Assessment of Iron Status at the Population Level; 2004 Apr 6-8; Geneva, Switzerland. Geneva: WHO; 2005.

11. Wheeler S. Assessment and interpretation of micronutrient status during pregnancy. Proc Nutr Soc. 2008 Nov;67(4):437-50.

12. Nemet D. Anemia and other manifestations of iron, vitamin B12 and folate deficiencies. Medicus. 2000;9(1):59-71. (In Croatian.)

13. Cook JD. Diagnosis and management of iron-deficiency anaemia. Best Pract Res Clin Haematol. 2005 Jun;18(2):319-32.

14. Scholl TO. Maternal iron status: relation to fetal growth, length of gestation, and iron endowment of the neonate. Nutr Rev. 2011 Nov;69 Suppl $1:$ S23-9.

15. Levy A, Fraser D, Katz M, Mazor M, Sheiner E. Maternal anemia during pregnancy is an independent risk factor for low birthweight and preterm delivery. Eur J Obstet Gynecol Reprod Biol. 2005 Oct 1;122(2):182-6.

16. Scholl TO, Hediger ML, Fischer RL, Shearer JW. Anemia vs iron deficiency: increased risk of preterm delivery in a prospective study. Am J Clin Nutr. 1992 May;55(5):985-8.

17. Shobeiri F, Begum K, Nazari M. A prospective study of maternal hemoglobin status of Indian women during pregnancy and pregnancy outcome. Nutr Res. 2006;26(5):209-13.

18. Scanlon KS, Yip R, Schieve LA, Cogswell ME. High and low hemoglobin levels during pregnancy: differential risks for preterm birth and small for gestational age. Obstet Gynecol. 2000 Nov;96(5 Pt 1):741-8.

19. Viteri FE, Berger J. Importance of pre-pregnancy and pregnancy iron status: can long-term weekly preventive iron and folic acid supplementation achieve desirable and safe status? Nutr Rev. 2005 Dec;63(12 Pt 2):S65-76.

20. Institute of Public Health Osijek-baranja county. Childbirths and abortions in healthcare institutions in Osijek-baranja county in 2009. Osijek (Croatia): Institute of Public Health Osijek-baranja county; 2010. (In Croatian.)

21. Institute of Public Health Osijek-baranja county. Childbirths in healthcare institutions in Osijek-baranja county in 2010. Osijek (Croatia): Institute of Public Health Osijek-baranja county; 2011. (In Croatian.)

22. Croatian Bureau of Statistics. Women and men in Croatia 2011. Zagreb: Croatian Bureau of Statistics; 2011.

23. Croatian Chamber of Medical Biochemists. Harmonization of laboratory analysis in the area of general medical biochemistry. Zagreb: Croatian Chamber of Medical Biochemists; 2004. (In Croatian.)

24. Andrews NC. Iron Absorption. In: Johnson LR, editor. Physiology of the gastrointestinal tract. Volume 2. 4th ed. Burlington: Elsevier; 2006. p. 1983-92.

25. Walsh T, O'Broin SD, Cooley S, Donnelly J, Kennedy J, Harrison RF, et al. Laboratory assessment of iron status in pregnancy. Clin Chem Lab Med. 2011 Jul;49(7):1225-30. 
26. Zilva JF, Pannall PR, Mayne PD. Clinical chemistry in diagnosis and treatment. 3rd ed. Zagreb: Školska knjiga; 1992. (In Croatian.)

27. Cameron BM, Neufeld LM. Estimating the prevalence of iron deficiency in the first two years of life: technical and measurement issues. Nutr Rev. 2011 Nov;69 Suppl 1:S49-56

28. Pasricha SR, Flecknoe-Brown SC, Allen KJ, Gibson PR, McMahon LP, Olynyk JK, et al. Diagnosis and management of iron deficiency anaemia: a clinical update. Med J Aust. 2010 Nov 1;193(9):525-32.

29. Rifas-Shiman SL, Rich-Edwards JW, Kleinman KP, Oken E, Gillman MW. Dietary quality during pregnancy varies by maternal characteristics in Project Viva: a US cohort. J Am Diet Assoc. 2009 Jun;109(6):1004-11.

30. Petrakos G, Panagopoulos P, Koutras I, Kazis A, Panagiotakos D, Economou A, et al. A comparison of the dietary and total intake of micronutrients in a group of pregnant Greek women with the Dietary Reference Intakes. Eur J Obstet Gynecol Reprod Biol. 2006 Aug;127(2):166-71.

31. Banjari I, Kenjeric D, Mandic ML. Iron bioavailability in daily meals of pregnant women. J Food Nutr Res [Internet]. 2013 [cited 2015 Mar 4];52(4):203-9. Available from: http://www.vup.sk/en/index.php?mainI $\mathrm{D}=2$ \&navID $=34$ \&version $=2 \&$ volume $=0$ \&article $=1890$.
32. Thompson B. Food-based approaches for combating iron deficiency. In: Kraemer K, Zimmermann MB, editors. Nutritional Anemia. Basel: Sight and Life Press; 2007. p. 337-58.

33. Rasmussen S, Bergsjø P, Jacobsen G, Haram K, Bakketeig LS. Haemoglobin and serum ferritin in pregnancy - correlation with smoking and body mass index. Eur J Obstet Gynecol Reprod Biol. 2005 Nov $1 ; 123(1): 27-34$

34. Källén $\mathrm{K}$. The impact of maternal smoking during pregnancy on delivery outcome. Eur J Public Health. 2001 Sep;11(3):329-33.

35. Langley-Evans SC. Metabolic programming during pregnancy: implications for personalized nutrition. In: Kok F, Bouwman L, Desiere F, editors. Personalized nutrition: principles and applications. Boca Raton: CRC Press/Taylor \& Francis; 2008. p. 101-14.

36. Godfrey KM, Forrester T, Barker DJ, Jackson AA, Landman JP, Hall JS, et al. Maternal nutritional status in pregnancy and blood pressure in childhood. Br J Obstet Gynaecol. 1994 May;101(5):398-403.

Received October 2, 2013 Accepted in revised form March 4, 2015 